\title{
RESEÑA ANALÍTICA COMO ESTRATEGIA DE ANÁLISIS DE TEXTOS
}

\author{
Oscar Manuel Ariza Orozco ${ }^{1}$ \\ Mariela Isabel Fuentes Pedrozo ${ }^{2}$
}

Fecha de Recepción: 1

Fecha de Aceptación: 3

SUMARIO: 1. Derecho del Espacio Ultraterrestre por Manfred Lachs; 2. Análisis de libro; 3. Datos generales de los reseñista; 4. Resumen del artículo o libro; 5. Glosario; 6. Contenidos: Nacimiento del Derecho Ultraterrestre; Cooperación Internacional; Principios Básicos del Espacio Ultraterrestre; Fronteras del Espacio Ultraterrestre; Situación Jurídica de los objetos lanzados al espacio ultraterrestre y los astronautas; Ayuda a los astronautas, devolución de astronautas y objetos espaciales; Telecomunicaciones en el espacio; Uso pacífico de armas y armamentos; Derechos y obligaciones de los Estados; Responsabilidad. Proceso legislativo; 7. Metodología; 8. Referencias bibliográficas.

${ }^{1}$ OSCAR MANUEL ARIZA OROZCO, Abogado titulado, Universidad Militar Nueva Granada, UMNG, Máster en Estudios Políticos Pontificia Universidad Javeriana, Especializado en Gestión Pública e Instituciones Administrativas, Universidad de los Andes. Docente investigador de tiempo completo, adscrito al departamento de Derecho Público y Derecho Internacional, Líder del grupo de investigación GruDPOE de la Facultad de Derecho y Ciencias Políticas, de la Universidad de Cartagena.

${ }^{2}$ Alumna de la asignatura de Derecho Internacional Público, V semestre período 2011.2, Facultad de Derecho y Ciencias Políticas Universidad de Cartagena. 


\section{COMO SE CITA ESTE ARTÍCULO (APA 6)}

Ariza Orozco O. M., Fuentes Pedrozo, M. I. (2014) Reseña analítica como estrategia de análisis de textos. (Y. Carrillo De la rosa, Ed.) Revista Jurídica Mario Alario D'Filippo, VII (11), pág. 134-150

\section{RESUMEN}

La reseña analítica es una estrategia metodológica para el análisis y comprensión de textos. A través de esta estrategia el estudiante, el investigador o el lector puede de manera ordenada profundizar sobre el contenido de un libro o texto escrito y así darle comprensión a la complejidad de su contenido. La aplicación de la reseña analítica es amplia ya que puede ser usada por estudiantes de pregrado, postgrados o investigadores y ayuda a la construcción de los marcos teóricos en investigación estricta o formativa. A continuación un estudiante y un docente comparten la aplicación de esta estrategia de estudio, analizamos un texto pertinente a la asignatura de derecho internación. El autor y título del libro es: Manfred Lachs, Derecho del Espacio Ultraterrestre, la ficha bibliográfica se puede ver a continuación al igual que el análisis y desarrollo de la reseña analítica.

\section{PALABRAS CLAVE}

Reseña analítica, análisis de textos, estrategias pedagogías, metodología del aprendizaje, investigación formativa.

\section{ABSTRACT}

The analytical review is a methodological approach for the analysis and comprehension. Through this strategy the student, the researcher or the reader can deepen orderly manner on the content of a book or written text and to give understanding of the complexity of its content. The application of analytical review is comprehensive as it can be used by undergraduates, postgraduates and researchers and help to build theoretical frameworks in strict training or research. Then a student and teacher share the application to be strategy study, we analyzed a relevant text on the subject of right hospital. The author and title of the book is: Manfred Lachs, Law of Outer Space, the bibliography can be seen below as well as the analysis and development of analytical review.

\section{KEYWORDS}

Analytical review, text analysis, strategies pedagogies, learning methodology, formative research. 


\section{DERECHO DEL ESPACIO ULTRATERRESTRE POR MANFRED LACHS}

\section{ANÁLISIS DE LIBRO}

Derecho del Espacio Ultraterrestre. Autor(es) del libro: MANFRED LACHS. Año de publicación: 1972. Editorial: Fondo de Cultura Económica. Ediciones F.C.E España S.A. ISBN: 84-375-0105-9.

\section{DATOS GENERALES DE LOS RESEÑISTA}

Pregrado: Derecho Asignatura: Derecho Internacional Público. Nombre del estudiante: Mariela Isabel Fuentes Pedrozo. Alumna de la asignatura de Derecho Internacional Público de $\mathrm{V}$ semestre de 2011, y Oscar Manuel Ariza Orozco, docente de la cátedra de Derecho Internacional Público, Facultad de Derecho y Ciencias Políticas, Universidad de Cartagena, Fecha de elaboración de la reseña: Noviembre de 2011.

\section{RESUMEN DEL ARTÍCULO O LIBRO}

En este libro, el autor desarrolla los avances del Derecho del Espacio Ultraterrestre. Comienza con un recuento histórico de cómo se dieron los primeros avances científicos y tecnológicos para que el hombre llegara al espacio ultraterrestre, luego desarrolla como nació el derecho espacial y los procesos para que se llegara a una regulación en este ámbito, los cuales en un principio se basaban en la analogía con otras ramas del derecho internacional, pero con el nacimiento de puntos problemáticos que no podían ser resueltos usando la analogía y se dio inicio al proceso legislativo en esta materia.

Además trata como se han abordado esos problemas, y como el derecho espacial se ha construido sobre las bases de igualdad y libertad de exploración y uso por parte de todos los Estados, inclusive aquellos que aun no han iniciado actividades espaciales.

También se trata en este texto la situación jurídica de los objetos espaciales y los astronautas, lo que se debe hacer en caso de accidentes espaciales, el proceso a seguir en caso de pérdida de un objeto o tripulación espacial y en caso de causar daños a terceros como determinar a los responsables para que se les indemnicen.

\section{GLOSARIO}

Autoridad de Lanzamiento: Es el Estado, organización intergubernamental o internacional, que es responsable del lanzamiento de un objeto.

Dstado de Lanzamiento: Es el Estado que lance o promueva el lanzamiento de un objeto espacial, o desde cuyo territorio o instalaciones se lance un objeto espacial.

$>$ Estado de Registro: Es un término utilizado para reconocer al Estado en el cual se encuentra registrado un objeto espacial. 
$>$ Perigeo: Punto de la órbita de la Luna o de un satélite artificial más cerca de la Tierra.

\section{CONTENIDOS}

El autor comienza planteando que el espacio es la parte constitutiva de cualquier sistema legal, y ese espacio con el desarrollo de las actividades humanas comenzó a expandir las dimensiones del Estado más allá de sus fronteras, en un principio solo era el suelo, luego el mar, el aire; con lo cual se tuvieron que dar nuevas regulaciones internacionales en material legal, en una primera etapa fueron bilaterales y luego multilaterales.

Nacimiento del Derecho Ultraterrestre: Con el desarrollo de actividades espaciales y la exploración en el espacio por parte del hombre, comenzó a surgir un vacío legal para regular las situaciones que pudiesen sobrevenir de las acciones realizadas en el espacio exterior. Por eso se necesito crear un nuevo sistema legal, pero no había bases para su creación, pues ninguna rama del Derecho Internacional se podía comparar con la rama naciente, por lo que se acordó que el espacio ultraterrestre podía ser explorado y usado por todos los Estados.

El Derecho Ultraterrestre se conformó principalmente por el Derecho Internacional y la Carta de la ONU -el cuerpo de la Carta, y las leyes y principios que se han ido agregando mediante resoluciones-.

La aplicación de la Carta y de los principios del Derecho Internacional no fueron inmediatos, pues parte de sus disposiciones eran para casos concretos y solo servían como base para el desarrollo posterior de los reglamentos especiales en el tema, eso sí teniendo en cuenta los principios básicos de la Carta, es decir, que todas las actividades que se fuesen a realizar debían tenar como fin la paz, la seguridad y la cooperación internacional.

A la hora de hacer extensiones del Derecho Internacional a esta rama surgieron inconvenientes, pues con las continuas exploraciones al espacio nacieron nuevos problemas que no podían ser resueltos, por ello los legisladores recurrieron a la analogía, para tratar de hacer una similitud con problemas resueltos anteriormente en otro campo para aplicarlo a la nueva rama. Esta analogía debía hacerse con cuidado, es decir, teniendo en cuenta el contexto para el cual se creó la ley y no repetir los errores que se cometieron.

Así como la analogía puede dar grandes beneficios, también puede perjudicar enormemente al desarrollo de un nuevo sistema, por lo tanto, si bien ayuda a dar soluciones a problemas que no se han resuelto, es necesario que se siga construyendo el nuevo sistema que tenga en cuenta reglamentaciones anteriores, pero que también innove y evolucione según las necesidades y los desarrollos recientes en su campo.

Cooperación Internacional: Legislar sobre las relaciones internacionales implica la cooperación de todos los Estados y de los organismos internacionales, buscando que las leyes satisfagan los intereses que hay en juego, no solo para las situaciones que ya han surgido, sino también para aquellas que estas apunto de surgir. 
Dada la importancia de este tema los Estados se colocan de acuerdo, para realizarlo bajo la protección de la comunidad internacional.

Los primeros pasos para la cooperación internacional se dieron en la década de 1950, cuando se estableció la Federación Internacional de Astronáutica, que luego se convertiría en la Academia Internacional de Aeronáutica establecida en 1960, en donde científicos de más de 66 países exploraban el espacio.

En un comienzo la cooperación fue tecnológica, y un ejemplo de ello fue la Conferencia Sobre la Exploración y Uso Pacífico del Espacio Ultraterrestre de las Naciones Unidas, en Viena en 1968, donde asistieron 78 países, 9 organismos especiales, 30 organizaciones internacionales gubernamentales y una organización no gubernamental. Los problemas que han surgido al legislar sobre el espacio ultraterrestre han sido impulsados a resolverse gracias a la labor de la Organización de las Naciones Unidas.

Cuando se obtuvo el éxito en el primer lanzamiento de un satélite, la ONU incluyó en su agenda los problemas del Espacio Ultraterrestre en 1957, y un año más tarde se presentó un proyecto sobre el Espacio Ultraterrestre ante la Asamblea General, entonces se estableció un comité ad hoc y dos años más tarde se convirtió en una comisión permanente. Luego en 1962 se creó la Subcomisión de Asuntos Científicos y Tecnológicos (encargada del intercambio y difusión de información sobre la investigación espacial), y la Subcomisión de Asuntos Jurídicos (Estudiar los problemas legales y pudieran surgir de la exploración del espacio ultraterrestre). Desde entonces la Comisión ha sido importante para la cooperación internacional.

Luego la Declaración de los Principios Legales que Rigen las Actividades de los Estados en la Explotación y Uso del Espacio Ultraterrestre, que fue adoptado por la Asamblea General el 13 de diciembre de 1963 y el Tratado de Principios Legales que Rigen las Actividades de los Estados en la Explotación y Uso del Espacio Ultraterrestre, incluso la Luna y otros Cuerpos Celestes, adoptado en 1966.

Principios Básicos del Espacio Ultraterrestre: el espacio por mucho tiempo fue olvidado por el hombre, hasta que Hugo Grocio se preguntó por él, con la aviación y el primer viaje de un satélite artificial nacieron cuestionamientos, sobre todo sí los Estados tenían soberanía sobre él y si era así como debían hacer para defender dicha soberanía, además como debía determinarse el espacio en el cual tenían que ejercer soberanía teniendo en cuenta el movimiento constante de la Tierra.

Por esos cuestionamientos se acordó que la Luna y otros cuerpos celestes, no pueden ser apropiados por una nación, ya sea por su uso y ocupación u otro medio, aun cuando algunos por uso entienden soberanía y "ocupación".

Con lo anterior se busca que todos los Estados puedan de igual manera explorar y hacer uso del espacio ultraterrestre, la Luna y los otros cuerpos celestes. Cumpliendo con los principios del Derecho Internacional que son principalmente la igualdad, la no discriminación y la libertad de exploración. Aunque no todos los Estados estén haciendo uso del espacio ultraterrestre, con estas 
reglas se busca proteger los intereses y derechos de los futuros Estados que quieran intervenir en su exploración; constituyéndose como límite de la libertad de exploración de los Estados lo derechos e intereses de los otros.

La libertad del espacio también incluye la libre investigación y todos los Estados pueden realizarla y con ella cooperar a la investigación internacional.

Muchos se preguntaron sobre qué se entiende por cuerpo celeste, pero no hay una medida física exacta para definir que es un cuerpo celeste, por lo tanto, son todas las aéreas territoriales del espacio ultraterrestre. Lo que indica que no pueden ser apropiados por ningún Estado, aun que si pueden ser explorados, usados e investigados libremente, y los resultados serán utilizados para la cooperación internacional y el desarrollo científico.

La libertad como principio del derecho del espacio ultraterrestre, implica la libertad de aterrizaje, exploración e investigación por parte de los Estado a los cuerpos celestes, y la igualdad al complementar este principio, conduce a deducir que ningún Estado puede negarle el acceso a otro para explorar un cuerpo celeste, por que más adelante se necesitaría una regulación más precisa para evitar futuros conflictos en caso de que algún país haya avanzado en la exploración de un cuerpo celeste no deje a otro ubicarse en el mismo lugar que él.

Por otro lado se ha preguntado la naturaleza jurídica del espacio exterior, algunos han dicho que es res extra commercium, res communis o res communis omnis, pero hay muchas dudas sobre sí los cuerpos celestes son res según la ley, por lo que la mejor solución es considerarlo como una esfera de actividad de los Estados con un régimen legal especial que goza de una protección particular.

Fronteras del Espacio Ultraterrestre: Teniendo en cuenta que el espacio ultraterrestre posee un status legal especial, ahora debemos preguntarnos ¿Cuáles son sus fronteras? Es por eso al decir, que el espacio ultraterrestre es un ambiente, entonces debemos determinar ¿cuáles son sus fronteras? que en este caso serian dos, una exterior y una interior. De la primera somos ignorantes y aparentemente no tiene fronteras, pues se cree que es infinito.

Entre tanto lo que llamamos frontera interior tiene otra situación, donde esta se confunde con el espacio aéreo. En un momento determinar la frontera no tenía ninguna importancia, hasta 1966 con la elaboración del Tratado del Espacio recobro relevancia.

Se buscaron y se presentaron muchas teorías para delimitar los dos ambientes, unos se basaban en la física o en la geofísica, otros en la propulsión de los vehículos espaciales, otros evocaban el control eficaz y algunos proponían una frontera específicamente definida. Finalmente algunos propusieron la naturaleza funcional como límite, es decir, las necesidades de los Estados en el espacio y el objeto en la línea divisoria. Con esta teoría se dieron diversas líneas divisorias, dependiendo de las actividades y la tolerancia de los Estados subyacentes.

Ahora hay que preguntarse ¿esa línea divisoria realmente existe? Revisando la validez de las propuestas anteriores llegaremos a una conclusión. En lo que respectas al ambiente, cada rama de 
la ciencia tiene distintos puntos de vista y aunque pudiese dividirse la atmósfera en capas es muy difícil su distinción, porque estas se unen, se mezclan y sufren fluctuaciones, además no son fácilmente visibles lo cual complica en gran modo la situación. La teoría que se basa en la propulsión de los vehículos tiene muchas fallas, pues la tecnología varia con el transcurso del tiempo y el alcance actual ira variando con los avances tecnológicos. Por lo cual, podemos afirmar que las dos anteriores teorías no nos ofrecen certidumbre, ni precisión ni vocación de permanencia.

En cuanto a la teoría del control de la eficacia, presenta muchos peligros por la forma y el grado de control, además los Estados con menor tecnología o sin potencial militar perderían sus derechos, favoreciéndose entonces a los Estados más poderosos.

Con lo visto anteriormente se hace necesario preguntar ¿si existe un dilema real? Para responder a esto se debe tener en cuenta el alcance de esta frontera, el valor y el verdadero interés en establecerla. En el primer aspecto, es solo un punto práctico y se reduce a una parte del espacio que se extiende sobre la Tierra y los mares de nuestro planeta. Esta es la zona entre el límite que alcanzan los aviones y el perigeo de una nave espacial que seguramente se irá anchando cuando los aviones alcancen más altura. El valor y el interés están vinculados a la soberanía de los Estados y con ello al concepto de seguridad nacional.

Teniendo en cuenta las teorías y la práctica, hay razones suficientes por la cuales no se puede delimitar la frontera entre el espacio aéreo y el ultraterrestre, pues estos son limitados por la soberanía de los Estados y no siempre las fronteras deben delimitarse, aunque para evitar conflictos, es mejor optar por delimitar una frontera convencional teniendo en cuenta el fin que persigue.

Como la frontera entre el espacio ultraterrestre y el aéreo no se ha determinado, cuando esta frontera se delimite, cualquier objeto que viaje de la Tierra al espacio exterior o viceversa, necesitara atravesar el espacio aéreo. Lo que no sería un problema si el objeto cae sobre el territorio del Estado que lo lanzó o sobre alta mar, el problema sobreviene cuando el objeto invade el espacio aéreo de otros Estados.

Como en un principio los Estados que practicaban estas actividades publicaban el lanzamiento, no informaban a los Estados afectados o no trataron de obtener permisos de los otros Estados involucrados; y a su vez los Estados afectados no protestaron, ni trataron de impedir estos vuelos, ni se reservaron el derecho de prohibirlo o no aclararon que en futuro requerirán de su consentimiento. Esto tal vez, se debió a que la práctica se inició en una época en el que el derecho ultraterrestre no estaba definido y se reconocía la libertad en el espacio exterior, y aunque no se puede denominar derecho de paso por el espacio aéreo, si ha continuado de este modo aunque ya existe una ley que regula ese tema.

Entonces por el silencio de los Estados afectados ¿se puede hablar de un derecho de vuelo establecido en el curso de los años?, este silencio lo que indica es el consenso sobre que el derecho de soberanía del Estado subyacente no ha sido vulnerado. 
Esta última posición es compatible con la teoría teleológica, es decir, que acepta el paso siempre que sea inofensivo para los Estados que conceden el paso, todo con el fin de explorar y usar libremente el espacio ultraterrestre. El paso inofensivo puede ser dado a todos los Estados, pero quienes lo conceden tendrían que renunciar a ciertas opciones importantes. Por lo tanto, se supone que el paso de objetos espaciales no les cause daños, pero como el paso es inofensivo y es una denominación muy amplia que debe definirse para evitar afectar al Estado subyacente.

Situación Jurídica de los objetos lanzados al espacio ultraterrestre y los astronautas: Con el paso del tiempo hay un gran número de objetos que se han lanzado al espacio ultraterrestre y aun no hay una clasificación de estos objetos, pues simplemente se les denomina «objetos lanzados al espacio ultraterrestre», tal vez su nombre es por temor a que cualquier clasificación quede obsoleta con el paso del tiempo, por ende, se debe proponer una clasificación amplia que pueda incluir cualquier objeto diseñado.

Dicha clasificación podría ser así: 1- para colocarse: a) En la órbita de la Tierra, de la Luna o de cualquier cuerpo celeste, o b) Sobre la Luna o cualquier cuerpo celeste. 2-Para seguir otro curso en el espacio ultraterrestre o a través de este. Esta clasificación aunque imperfecta serviría de guía útil para la aplicación de las leyes existentes. Y esto conlleva a reflexionar sobre dos problemas diferentes, que son la jurisdicción y la propiedad.

La jurisdicción es atributo básico del Estado, por medio de la cual ejercita sus poderes como sujeto del Derecho Internacional, estos poderes no son ilimitados y encuentran su límite en los derechos de otros Estados y la necesidad de cooperación en las relaciones internacionales. Desde el punto de vista del Derecho del espacio ultraterrestre, solo se puede hablar de ejercer jurisdicción sobre los objetos espaciales y sobre el personal que lo ocupa.

Sobre los objetos y el personal, la jurisdicción y el control lo posee el Estado en el cual el objeto se encuentra registrado, y por lo tanto, esto implica que este Estado está a cargo de la seguridad del personal, que los objetos o el personal no infrinjan los derechos de los otros Estados y que la misión no viole las disposiciones legales del espacio ultraterrestre.

El principio adoptado para los objetos espaciales no se basa en ley de bandera, ni en el concepto de nacionalidad, sino que se basa en la jurisdicción, y su ejercicio se vincula a dos elementos esenciales: el registro y la situación del objeto en un medio definido.

El registro de la Secretaria General de la ONU es diferente, pues del registro que se habla es el que cada Estado debe establecer y mantener, siendo esto importante para la identificación de objetos que causan daños. El Estado de registro hoy identificado como Estado de lanzamiento, tienen sus diferencias, el último es el Estado que lance o promueva el lanzamiento, o desde cuyo territorio o instalaciones se lance un objeto espacial. Mientras que el primero es el Estado donde se encuentra registrado un objeto espacial.

No existe problemas si un Estado realiza las dos actividades, el conflicto es cuando hay varios 
Estados involucrados y ellos deberán acordar cual será el Estado de registro, y en un principio será quien ejerza jurisdicción sobre él.

El status del objeto y el status del personal están interrelacionados. En el primero la jurisdicción se extiende sobre todo el objeto o las partes de este que se haya separado, en todo caso aunque se una a otros objetos y formen una unidad se conserva la jurisdicción.

El status del personal hace referencia a los individuos "astronautas» se pregunta ¿a quienes incluye? ¿Sí a todos los de la tripulación? aun cuando hoy solo se puede hablar de este concepto en el futuro pueden existir pasajeros, y si los hay darles el mismo status es lo lógico. Pero sea cual fuere su nacionalidad, permanecen bajo la jurisdicción del Estado en cuyo registro figura el objeto en que viajan.

Sí el lanzamiento es un conjunto entre varios Estados pueden convenir que la jurisdicción la tenga un Estado diferente al de registro, e igualmente continuarán bajo su jurisdicción aunque entren en una instalación de jurisdicción de otro Estado.

Como en todo viaje puede haber tropiezos y el aterrizar en otro Estado diferente por una emergencia, en este caso la jurisdicción es solo para cuando se esté en el espacio ultraterrestre, entonces deben regularse estas situaciones, y un modo de ver esa incursión es como una paso inofensivo, y el Estado donde aterrice de emergencia le debe garantizar a los tripulantes un seguro y rápido regresos al Estado de lanzamiento o de registro, y así el Estado subyacente no podría reclamar la jurisdicción sobre la tripulación.

En lo que respecta a la propiedad, aunque el espacio ultraterrestre, la Luna y otros cuerpos celestes no pueden ser objeto de apropiación, los objetos lanzados si son susceptibles de propiedad y es protegida en cada etapa de su viaje y en todos los ambientes.

Los objetos espaciales pueden ser reclamados o restituidos, sí caen en un territorio diferente, y ese derecho de pertenecer al Estado de registro. Inclusive si se abandonan, pueden constituir una res nullius, pero el Estado de registro conserva las obligaciones que resulten de su lanzamiento y sus consecuencias. Estos objetos pueden cambiar de dueños y si eso sucede, se deben hacer los cambios correspondientes en el registro.

Ayuda a los astronautas, devolución de astronautas y objetos espaciales: los viajes espaciales implican riesgos por ello es necesario la cooperación internacional, para ayudar a los individuos en riesgo en los accidentes y en el rescate de objetos y de personas.

Por eso, se han establecido unos principios que se deben garantizar como: 1) La ayuda a los astronautas en caso de accidente, peligro o aterrizaje forzoso, 2) su regreso seguro y pronto, 3) la colaboración entre astronautas de diferentes Estados. Estas disposiciones aplican para los astronautas como para las personas que estén en la nave. 
Las ayudas deben ser prestadas ya sea en el territorio donde un Estado ejerce jurisdicción o en un territorio sin jurisdicción o en altamar.

Cuando un Estado recibe información o descubre que ha ocurrido uno de estos sucesos debe notificar a la autoridad de lanzamiento, sino la conoce debe dar aviso público y notificar a la Secretaria General de la ONU.

El Estado que ejerce jurisdicción sobre el territorio en que el personal de una nave espacial tiene un aterrizaje forzoso o aterriza por accidente, debe prestarle toda la ayuda requerida y notificar a la autoridad de lanzamiento y al Secretaria General de la ONU las medidas adoptadas. Si no posee los medios necesarios solicitará la colaboración de las autoridades de lanzamiento, para realizar las maniobras de ayuda correspondiente a la tripulación, la autoridad de lanzamiento no puede negarse a prestar la ayuda requerida.

Si la tripulación desciende en altamar o en un lugar sin jurisdicción de un Estado, el Estado que primero reciba la información de lo ocurrido esta obligado a prestar ayuda para la búsqueda y rescate. Dicha colaboración solo es obligatoria si es necesaria y sí se cuenta con los medios para ello, en caso de participar debe informar a la Secretaria General de la ONU. La obligación de ayuda mutua incluye a los astronautas en el espacio ultraterrestre y cuerpos celestes.

Luego de efectuado el rescate, el Estado que haya llevado a cabo el salvamento debe ofrecer todas las garantías a los rescatados para que retornen de manera segura y rápida a su lugar de origen.

Hay problemas similares cuando es un objeto, así que el Estado que sepa que ha caído sobre su territorio, en alta mar o en un territorio sin jurisdicción estatal deberá informar a las autoridades de lanzamiento y a la Secretaria General de la ONU. El Estado donde caiga dicho objeto solo lo rescatará sí se lo solicitan y solo prestará las medidas que pueda prestar, y en caso de necesitar ayuda puede solicitarla a la autoridad de lanzamiento. Si el objeto cae sobre un lugar sin jurisdicción su rescate será de manera voluntaria.

Los objetos lanzados que a su regreso a la Tierra sean un riesgo o sean nocivos para la vida y salud, o dañosos para el ambiente, las autoridades de lanzamiento deberán tomar medidas inmediatas y eficaces para eliminar el posible riesgo.

Para la devolución de los objetos espaciales recuperados, primeramente se debe enviar una solicitud de devolución por parte del Estado que lo lanzó, luego el Estado que lo tiene deberá restituírselo o ponerlo a disposición de las personas autorizadas para recogerlo.

Los gastos generados de la recuperación, el rescate y devolución de astronautas y objetos espaciales, que hace un Estado que presta la ayuda, serán reembolsados por parte de la autoridad de lanzamiento.

Telecomunicaciones en el espacio: las telecomunicaciones en el planeta son muy importantes y con el desarrollo de las actividades espaciales han tomado una mayor importancia, además se han 
dado innovaciones tecnológicas en este campo que amplían la cobertura y las funciones de los satélites, por lo cual, se hace necesario que exista una ley de coordinación entre los Estados para divulgar la información obtenida, para que sea utilizada en beneficio de todos, y que garantice el libre acceso de todos a los medios de comunicación espacial.

Se temía que las imágenes y sonidos emitidos pudiesen ser usados por inescrupulosos que sin autorización emitan mensajes que pongan en peligro la seguridad y la paz mundial, por ello, estas actividades deben tener como fin primordial la paz y la cooperación internacional, según lo dicho en el Tratado del Espacio y en las Resoluciones de la ONU sobre este tema.

Uso pacífico de armas y armamentos: con la apertura de espacio ultraterrestre, nacieron preocupaciones sobre la posible competencia bélica y armamentista que pudiesen iniciar los Estados, colocándose en peligro la paz. Previendo dicha situación, la Asamblea General de la ONU fomentó el uso pacífico del espacio ultraterrestre, sin embargo, muchos interpretaron que el uso pacífico excluía la agresividad entre Estados, pero no el uso militar del espacio ultraterrestre. Otros por su lado, aducen que sí la intensión era prohibir su uso agresivo, solo habrían tenido que remitirse al Derecho Internacional y a la Carta de la ONU.

Por ese dilema, se aclaró que en el espacio ultraterrestre queda prohibida la instalación de bases militares, el ensayo con armas o la realización de maniobras militares, con esto se busca proteger la paz y que el espacio ultraterrestre solo sea usado para la cooperación internacional, la investigación yexploración.

Ahora la desmilitarización del espacio y los cuerpos celestes, no prohíbe el uso de personal militar, pero su empleo solo se dará si es necesario para la investigación o cualquier otra finalidad pacifica.

Derechos y obligaciones de los Estados: el uso del espacio ultraterrestre lleva consigo un conjunto de derechos y obligaciones, donde los principales sujetos son los Estados, que actúan por si mismos o a través de una organización gubernamental o no gubernamental, o por personas jurídicas o naturales. Sin embargo, se han concedido funciones a los organismos internacionales.

Algunas de las obligaciones de la ley espacial están dirigidas a los Estados, pero otras son erga omnes. Entre esas obligaciones están: a) Los Estados deberán informar a otro Estado o a la Secretaria General de la ONU, sobre cualquier fenómeno que descubran durante la exploración o investigación científica que pueda constituir un peligro para la vida y salud de los astronautas; b) la obligación de los Estados de permitir el acceso a otros Estados a sus vehículos espaciales, instalaciones, estaciones o equipo que este en el espacio ultraterrestre, siempre que el Estado que quiera acceder a ellos debe informar con un tiempo razonable la visita que pretender realizar; c) la obligación de los Estados de considerar las peticiones para observarlos vuelos de objetos espaciales que ellos lancen; d) la obligación de cualquier Estado de consultar sí al iniciar una actividad o experimento en el espacio pueda tener consecuencias en el ambiente; e) informar a la Secretaria General de la ONU, al público y las organizaciones científicas la naturaleza, la realización, localización y resultados de actividades espaciales. 
Dichas obligaciones buscan eliminar la disparidad en la capacidad científica y tecnológica existente entre los Estados, garantizándose la igualdad entre los para explorar el espacio ultraterrestre y evitar que un Estado se otorgue derecho exclusivos y usarlo en detrimento de otro Estados.

Responsabilidad: Al los Estados practicar actividades espaciales contraen derechos y obligaciones, con lo que también una extensión de responsabilidad.

La responsabilidad que nace de las actividades espaciales tiene un campo amplísimo, pues dichas labores pueden poner en riesgo desde una persona hasta toda la humanidad entera, por lo tanto no se pueden abarcar todos los casos específicos, sino que se da un primer paso para su reglamentación y los rasgos generales en los cuales un Estado debe responder.

Lo primero es resaltar que los responsables en un primer lugar son los Estados, ya sea que hayan actuado por si mismos o a través de una organización no gubernamental, ellos responden por el daño causado a otro Estado, a otras personas ya sean naturales o jurídicas. Al ser los Estados quienes respondan por sus actividades en el espacio ultraterrestre se garantiza el cumplimiento de la ley espacial. Por consiguiente, los Estados deben tomar las medidas necesarias para asegurar que las personas naturales y jurídicas que se dedican a estas actividades cumplan con la normativa internacional.

Para determinar al responsable de un daño, se ha fijado el siguiente criterio: es responsable el Estado que lance o intente lanzar un objeto espacial, o desde cuyas instalaciones es lanzado. Cuando dos o más Estados lanzan conjuntamente un objeto espacial, son responsables mancomunada y solidariamente de cualquier daño causado.

Aunque los Estados son los sujetos principales de derechos y obligaciones, esto no excluye que puedan actuar a través de una organización internacional. En este caso la responsabilidad recae sobre la organización y los Estados miembros, la organización y los Estados miembros son responsables mancomunada y solidariamente. Sin embargo, primero debe reclamarse a la organización y si esta no cumple se reclama a los Estados.

Ahora para tasar el monto de la indemnización por el daño se debe actuar en equidad y justicia, y determinar económicamente cuánto costaría resarcir el daño causado, de tal manera que se restituya al Estado, persona natural o jurídica, u organización internacional a la situación que habría existido de no haberse causado el daño.

Cuando un Estado sufra un daño por un objeto espacial y no se conozca quien lo lanzó, se buscará la asistencia equitativa y razonable para la identificación del objeto.

Proceso legislativo: El proceso de formación de la ley espacial inició con el envió de objetos al espacio, con ello se reconoció que debía regularse sobre esta materia y así el Derecho Internacional entraría a esta nueva dimensión. 
En los comienzos la regulación era modelada por la práctica, pero como esta situación no podía dejarse de este modo, comenzaron a surgir iniciativas para la creación de una ley escrita.

Dicha tarea se le encargó a las Naciones Unidas y como estaba claro que no podía adoptarse un documento estático, se acordó que se prestaría a la evolución y a la adopción gradual de principios y normas legales, así se estableció una comisión espacial para que se encargara de trazar los principios de la ley. Luego de aprobados en la comisión eran llevados a la Asamblea General, después de allí aprobados se remitían a la plenaria para ser votados.

El proceso legislativo tuvo dos etapas, en la primera se dieron una serie de resoluciones por parte de la Asamblea General sobre la cooperación internacional y los principios básicos del espacio ultraterrestre, la primera etapa culminó en 1963 con la Declaración de los Principios Jurídicos que Rigen las Actividades de los Estados en la Exploración y Uso del Espacio Ultraterrestre.

Se inició la segunda etapa en 1967 cuando se concluyó el Tratado de los Principios que Rigen las Actividades de los Estados en la Exploración y Uso del Espacio Ultraterrestre, incluso la Luna y otros Cuerpos Celestes. Esta no era solo una declaración que confirmaba los principios adoptados anteriormente, sino que avanzó y enriqueció la ley con nuevas disposiciones importantes y sustanciales. Pues el tratado ayudó a disipar ciertas dudas que existían con el uso del espacio ultraterrestre.

Luego de elaborado el tratado la Subcomisión de Asuntos Jurídicos se dedicó a elaborar instrumentos específicos y así se redactó el Acuerdo sobre Rescate de Astronautas, Regreso de los Astronautas y Devolución de los Objetos Lanzados al Espacio Ultraterrestre, que fue aprobado en diciembre de 1968; a este acuerdo le siguió la elaboración del Convenio sobre Responsabilidad, el cual fue aprobado en 1971, luego en 1974 se aprobó el Convenio sobre el Registro de los objetos lanzados al espacio ultraterrestre.

La tarea de legislar las primeras normas sobre el uso del espacio ultraterrestre aunque fue larga, ardua y metódica conto con la participación de toda la comunidad internacional y abrió camino para las futuras regulaciones sobre el tema.

\section{METOdOLOGÍA}

El autor ha desarrollado el documento de manera expositiva planteando todo lo que ha sido el nacimiento y desarrollo del Derecho Espacial, además expone los principales temas que se regularon en el uso del espacio ultraterrestre haciendo uso de los convenios, tratados, resoluciones, avances y comentarios hechos sobre la materia tratada. 


\section{REFERENCIAS BIBLIOGRÁFICAS}

ABRAHAM, H. J. (1960) “Das Recht der Luftfarth”. Colonia- Berlín, 2 vols.

ADAMS, T.R. (1968) "Outer Space Treaty: An Interpretation in Light of the No. Sovereingnty y Provesion". 9 Harvard International Law Journal, pp.140y ss.

BAKER, A.S. (1966) “Military Implications of Space, I.C.L.Q”. Publicación especial, n.9, pp. 73 y ss.

BASKIN, I. y A. (1968) “Problemas jurídicos de las interferencias en la atmósfera (en ruso)”. Pravovedenei, n.1, pp. 103 yss.

BIERZANEK, R. (1961) “La natura juridique de la haute mer, R.GD.I.P”. págs. 233 y ss.

BRIKELY, J. L. (1963) “The Law of Nations”. 6 edición. Oxford.

BUSTAMANTE Y SIRVEN, A.S. (1930) “La mer territoriale”. París.

CASTAÑEDA, J. (1969) “Legal Effects of United Nations Resolutions”. Nueva York.

COHN, G. “La Théorie de la responsabilité internationale”. 68 R.A.

CREBOLA, P. (1967) "Raumfahrt und Völkerrecht”. Zürich.

DAHM, G. (1958) “Völkerrecht”. Stuttgrat.

DUTHEIL DE LA ROCHERE. J. (1967 “La Convention sur l'internationalisation de l'espace”. 13 A.Fr.D.I., pp. 607 yss.

FENWICK, G.G. (1958) “How High in the Sky”. 52 A.J.I.L., pp. 96 yss.

GAL, G. (1959) “Space Law”. Budapest.

GOLOVIN, M.N. (1962) “Confilct in Space”. Londres.

HALL, R.C. (1969) “Rescue and Return of Astronauts on Earth and Outer Space”. 63 A.J.I.L, pp. 197 y ss.

JACOB, N.H. (1952) “Droit interplanétaire”. 15 Revue générale de l'Air, págs. 287 y ss.

KUZNETSOV, B.G. (1969) “Nauka w 2000 godu (la ciencia en el año 2000)”. Moscú.

LARSEN, P.B. (1966) “A simple of Space Law Opinion”. 27 Ohio State Law Journal, n.3 pp. 468 y ss.

McNAIR. (1953) "The Law of the Air". London.

MOUNTON. M.W. (1962) “The International Regime of the Polar Regions”. 107 R.C.A.D.I., III, pp. 175 Y ss.

NICOLSON, M. (1970) “The Environmental Revolution”. Londres.

PATTERSON, E.W. (1963) “Law in Scientific Age”. Nueva York. 
REINTANZ, G. (1967) “Weltraumrecht”. Berlín.

SMEDAL, M. (1931) “Acquisition of Sovereingnty over Polar Areas”. Oslo.

TAYLOR, G.A. (1970) “The Doomsday Book”. Londres.

VALLAT, F. (1969) "The Outer Space Treaty". 73 The Aeronautical Journal, pp. 754 yss.

WENGLER, W. (1964) “Völkerrecht”. Berlín. 2 vol.

ZYLICZ,M. (1958) “Sur quelques problemas de droit astronautique”. R.G.D.I.P., pp. 655 y ss.

\section{CITAS TEXTUALES:}

a) "De acuerdo, con el derecho internacional, incluida la Carta de las Naciones Unidas, para mantener la paz y la seguridad internacionales y para promover la cooperación y el entendimiento internacionales". Art.3 del Tratado que Rige las Actividades de los estado en la Exploración y el Uso del Espacio Ultraterrestre.

b) “El espacio ultraterrestre, incluso la Luna y otros cuerpos celestes, no pueden someterse a una apropiación nacional reclamando al soberanía, por medio del uso o la ocupación, ni por ningún otro medio". Art.2 Tratado de los Principios que Rigen las Actividades de los Estados en la Exploración y Uso del Espacio Ultraterrestre, incluso la Luna y otros Cuerpos Celestes.

c) "El espacio ultraterrestre, incluso la Luna y otros cuerpos celestes, podrán ser libremente explorados y usados por todos los países sin discriminación de ningún tipo, basándose en la igualdad y de acuerdo con el derecho internacional". Art.1, párrafo 2 del Tratado del Espacio de 1967.

d) "Realizaran todas sus actividades en el espacio exterior, incluso en la Luna y otros cuerpos celestes, con el debido respeto a los intereses de todos los demás Estados". Art. 9 del Tratado de 1967.

e) “En cuyo registro figura el objeto lanzado al espacio ultraterrestre, retendrá su jurisdicción y control sobre tal objeto y del personal que en él hubiere mientras se encuentre en el espacio ultraterrestre o en un cuerpo celeste". Art. 8 del Tratado del Espacio de 1967.

f) “El interés común de la humanidad en fomentar el uso pacífico del espacio ultraterrestre y la urgente necesidad de vigorizar la cooperación internacional en este importante campo". Resolución de la Asamblea General 1721 (XIV) del 12 de diciembre de 1959.

g) “Contribuir a una amplia cooperación internacional en los aspectos científicos y jurídicos de la exploración y el uso del espacio ultraterrestre para fines pacíficos" Tratado del Espacio, 1967, cuarto párrafo del Preámbulo. 
h) "La Luna y otros cuerpos celestes se usarán exclusivamente con fines pacíficos. Está prohibido establecer bases, instalaciones y fortificaciones militares, ensayar cualquier tipo de armas y realizar maniobras militares en los cuerpos celestes" Art 4. Párrafo 2 del Tratado del Espacio, 1967.

i) "No recibir de ningún donador armas nucleares y otros artefactos nucleares explosivos, ni aceptar, directa o indirectamente, el control de estas armas o artefactos explosivos; no fabricar o adquirir armas nucleares $u$ otros artefactos explosivos nucleares; y no buscar o recibir ninguna ayuda en la fabricación de armas nucleares o de otros artefactos explosivos nucleares". Art. 2 del Tratado de Proliferación de las Armas Nucleares, 1 julio de 1968.

j) “ No transferir a ningún país ningún tipo de armas nucleares u otros artefactos explosivos nucleares, ni ceder el control directo o indirecto de estas armas o artefactos explosivos, y no ayudar de ninguna manera, alentar o inducir a ningún Estado que no tenga armamentos nucleares a fabricar o a adquirir de alguna otra manera armas nucleares o otros artefactos explosivos nucleares, o tener el control de estas armas o artefactos explosivos" Art.1 del Tratado de Proliferación de las Armas Nucleares.

k) “Inter alia cualquier medida de carácter militar, como establecer bases y fortificaciones militares, efectuar maniobras militares, y también ensayar cualquier tipo de armas" Tratado del Antártico de 1959. Art.1. Párrafo 1.

I) "Donde no existe delito ni omisión de un deber no pude haber nada para apoyar un cargo de responsabilidad o justificar una demanda" El caso de Jamaica, Comisión de Reclamaciones de Inglaterra- Estados Unidos 1789, Moore, Adjudicaciones internacionales, Serie moderna, IV, p.499.

m) “Cualquier persona que sufra daño sobre la superficie de la Tierra, solamente aportando pruebas de que el daño fue causado por un avión en vuelo o por cualquier persona o cosa que caiga de este, tiene derecho a una indemnización..." ICAO, Roma 1962, Art.1, párrafo 1.

n) "Si el daño es causado por un objeto espacial representa un peligro a gran escala para la vida humana o repercute gravemente en las condiciones de la vida de la población o de funcionamiento de los centros vitales, los Estados miembros, y en particular el Estado que lanza el objeto, examinarán la posibilidad de prestar ayuda rápida y adecuada al Estado que ha sufrido el daño, cuando este así lo solicite".

o) “En este sentido y en otro más amplio debe quedar claro que los principios enumerados no constituyen un capítulo ya terminado. Debemos estar satisfechos de lo que se ha logrado y esforzarnos por conseguir nuevos acuerdos. La ley del espacio ultraterrestre solo se encuentra en su etapa de formación. Debemos actuar con prudencia y cuidado, aprovechar plenamente los acuerdos alcanzados, hacer de ellos una realidad viviente y continuar nuestros esfuerzos por realizar otros convenios. El proyecto adoptado por la Asamblea General puede y debe convertirse en un documento de importancia básica en nuestros futuros esfuerzos, para facilitar la cooperación internacional, para regular y ofrecer protección legal al logro del genio humano en el espacio ultraterrestre para el beneficio de nuestra generación y de las generaciones que vendrán después de nosotros" Asamblea General, registros oficiales, XVIII, anexos, punto 28 , pág. 15.

p) "Los tratados han sido, son y seguirán siendo los instrumentos más eficaces por lo que los Estados adquieren derechos y obligaciones en sus relaciones mutuas. Algunos son fundamentos para el desarrollo de las relaciones internacionales. Por ello, era evidente y lógico que se concluyera un 
tratado sobre esta materia, un tratado universal en su alcance y en su carácter" Primer Comité de la Asamblea General, 17 de diciembre de 1966.

q) "La ley del espacio ultraterrestre ha sido modelada con toda la cooperación de la comunidad internacional. Este hecho fue muy alentador, ya que, en la práctica, el espacio ultraterrestre solo era explorado y utilizado por un pequeño número de Estados" Dr. Krishna Rao.

\section{COMENTARIO PERSONAL Y CONCLUSIONES:}

Conclusiones: Si bien el Derecho espacial ha conseguido grandes avances, con el desarrollo de nuevas tecnologías TICs, se tendrán que crear nuevas normativas para regular nuevas situaciones que se irán presentando con el transcurso del tiempo, por ello las bases sentadas para la creación de una nueva normativa no son estáticas, sino que pueden acomodarse según las situaciones y problemas que se presenten.

Por ello la tarea del Derecho Internacional en esta materia debe ser constante y contar con la cooperación de la sociedad internacional para elaborar las nuevas disposiciones, que deben ser hechas con una visión futurista, pues el hombre cada día es más curioso y posa su mirada en el espacio exterior y con ello desarrolla tecnologías para perfeccionar la exploración, uso e investigación del espacio ultraterrestre. Si no se hace de esta forma el Derecho Espacial estaría condenado a quedar rezagado y con grandes vacios jurídicos sino va regulando al paso que van surgiendo las nuevas problemáticas en este campo.

Comentario: el texto es una fuente importante para conocer los gérmenes del Derecho espacial y si bien tiene años de haberse escrito muchos de los puntos y recomendaciones hechas por el autor tienen vigencia y sirven como base para la elaboración de las nuevas directrices sobre el espacio ultraterrestre.

El autor deja una posición muy bien sentada al decir que es un derecho que va unido indefectiblemente con la tecnología y que no se puede crear una legislación sobre el tema atada a las tecnologías existentes en el momento de su expedición, pues estaría condenada a quedar obsoleta en muy poco tiempo, es por eso que es recomendable elaborar estas normas del modo más amplio posible.

Para algunos autores ascender al espacio ultraterrestre no solo se requiere de un desarrollo científico y tecnológico, sino la seguridad por causa del uso de vehículos espaciales tripulados y no tripulados. El otro aspecto es en relación con la similitud que hacen de de altamar o mar de todos y el espacio ultraterrestre a los cuales todos los estados tienen uso y acceso "libremente".

La creación e inicio de los vuelos espaciales desde y la fundación de las dos más grandes agencias científicas como la NASA de los Estados Unidos de Norteamérica, el 29 de julio de 1958 el presidente Dwight David Eisenhower firmó el acta que fundó la NASA, la cual empezó a funcionar el 1 de octubre de 1958 con cuatro laboratorios y unos 8 mil empleados. La URSS inicio la exploración espacial comenzando con el lanzamiento del satélite artificial Sputnik el 4 de octubre de 1957, en el Cosmódromo de Baikonur (base de lanzamiento de cohetes de la URSS), estos dos países fueron los pioneros y han impulsado científica y tecnológicamente la exploración del espacio aéreo, los viajes tripulados y no tripulados, la construcción de estaciones espaciales el viaje no tripulado a Marte y sobre todo el descubrimiento de elementos que soslayan la posibilidad de vida en otros confines del universo distintos al planeta tierra. 